investigation, in Dr. Langmuir's view, confirm many features of the structure to which the theory had led.

In the discussion which followed the address, Dr. Wrinch explained that the method used by Langmuir and Wrinch in interpreting the vector maps was a geometrical formulation of the classical picture of a crystal as a set of discrete units. Prof. J. D. Bernal uttered a word of caution against supposing that the theory was proved. He thought that certain features must be true, and others were possibly true, but in his view the X-ray data fail to confirm the proposed structure. $\mathrm{He}$ had had a vector map calculated for the latter, and found that it differed very markedly from that obtained experimentally. Prof. E. H. Neville showed the care that is needed in interpreting a vector map, by a simple example in which a map constructed from a given set of points appeared at first sight to correspond to quite a different type of distribution.

\title{
Studies in Galactic Structure
}

$\mathrm{I}^{\mathrm{N}}$ Bulletin of the Astronomical Institutes of the Netherlands, No. 308, J. H. Oort investigates the structure of the galactic system from counts of faint stars in medium and high galactic latitudes combined with absorption data from Hubble's counts of extra-galactic nebulæ. Data for the star density were derived from Kapteyn's Selected Areas; the amount of absorption to be used for each selected area was estimated from nebular counts in neighbouring fields.

Assuming tentatively that the absorption occurs in front of the stars studied, the investigation leads to a picture of the galactic system differing somewhat from the prevalent conception : broadly, the sun appears to be situated in a region of relatively low star density surrounded in all galactic longitudes by extensive regions where the density is at least twice as high. The structural features indicated are large-scale phenomena, extending on both sides of the galactic plane to distances of 500 or 700 parsecs (say 1600 2300 light years) from this plane. The analogy between this structure and the spiral structure of strongly flattened extra-galactic systems is discussed; but the data are insufficient to define the course of the spiral arms, although the sun apparently lies between two arms. At greater distances from the galactic plane, the unevenness in density distribution disappears; at levels between 800 and 1800 parsecs the equidensity surfaces are satisfactorily represented by planes inclined at angles of about $10^{\circ}$ to the galactic plane, implying an increase of density in the direction of the galactic centre by a factor $\mathbf{1} \cdot 38$ per 1000 parsees. The derived longitude of the centre is $324^{\circ} \pm 3^{\circ}$ (region of Scorpio-Sagittarius), in excellent agreement with the longitude derived from the effects of differential rotation or from the distribution of globular clusters and planetary nebulæ. As regards selective absorption, the evidence from colour-excess data in the Kapteyn Areas points to a strong galactic concentration of the absorbing clouds, and indicates that the greater part of the absorption derived from Hubble's counts of nebulæ must have occurred relatively close to the galactic plane.

From the time of Sir William Herschel, the dark patches and lanes, more or less devoid of stars, in the Milky Way have been an attractive problem for astronomers. It is now known that these dark areas must be due chiefly to clouds of cosmic dust. In Publication No. 48 of the Kapteyn Astronomical Laboratory at Groningen, an estimate is made by B. Hiemstra of the distances of some absorption clouds in areas 2, 5, 9 and 24 of Kapteyn's Selected Areas.

From a statistical study of proper motions and apparent magnitudes of the stars in the absorbing regions and in the surrounding rich areas, the author is able to derive limits for the distance and absorption effect of the clouds, assuming them to be of infinitesimal thickness compared with their distance. The results range as follows: Area 5 contains a cloud absorbing $0 \cdot 8^{\mathrm{m}}-1 \cdot 5^{\mathrm{m}}$ at a distance less than 300-400 parsecs. Area 2 contains a cloud absorbing at least $0 \cdot 5^{\mathrm{m}}$ at a distance of $600-1200$ parsecs. In area 9, lying east of the North America Nebula, the absorption reaches $2 \cdot 0^{\mathrm{m}}$ at a distance of 500-650 parsecs. The plates used in the investiga. tion were taken at the Radcliffe Observatory, Oxford, with the 24-inch refractor, which was recently presented to the University of London Observatory at Mill Hill. Usually the pairs of plates were taken about fifteen years apart.

\section{Education for Citizenship}

DREPARATION for and training in citizenship are recognized by an increasing number of people in Great Britain as of vital national importance and as calling urgently for the development and extended application of effective techniques. There is much to interest them in "Forums for Young People", a pamphlet recently issued by the United States Office of Education (Washington, D.C., Superintendent of Documents. 15 cents). This is a study of problems and plans involved in providing organized series of free discussions of public affairs for high school and college students and for other young people. For two years, the Commissioner of Education has conducted, with the help of very substantial Federal grants, a vigorous propaganda for the organization of public affairs forums throughout the States, and the resulting experiments have provided ample material for this study, the publication of which will, he hopes, "contribute to the thinking of the teaching profession and of laymen on this problem of finding ways and means to make secondary and higher education meet its civic responsibilities". It begins with an admirably lucid discussion of pros and cons. The point of view taken is that the most important purpose of education in our time is the training of the mind to deal intelligently with the undecided issues which face mankind. Subsequent chapters describe: types of forum 
programmes for high schools, forum plans for colleges and universities, forum plans for youth on community-wide basis and "guide-posts to organisation".

Some of the problems of "Forums for Young People" were discussed at the Secondary School Conference held by the Association for Education in Citizenship last year at Morley College, London. A report of the proceedings at this conference appeared in the July number of the Citizen. The conference covered a wider field, including consideration of the part played by character and purpose in the making of good citizens, and the efficacy of various out-ofschool activities such as scouting, classes in first-aid, and camping as means for stimulating emotion and imagination and harnessing them to action in the service of the community so as to overcome "that apathy which is the besetting weakness of democracy". With the view of keeping in touch with one another the now considerable number of schools where systematic efforts are made to foster the development of a spirit of community service a "Schools Community Service Group" has been constituted within the Association with headquarters at
The Downs School, Colwall, Malvern, with Mr. W. F. Hoyland as honorary secretary. The chief subjects dealt with by the group are : manual work on the school estate, help at physical training and play centres, boys' clubs and school missions, visits to factories, housing estates, etc., work camps in the holidays, for example, helping unemployed men on their allotments, school expeditions abroad.

In the same number of the Citizen is an article of outstanding interest by Prof. René Maheu of the Institut Francais du Royaume-Uni on the teaching of philosophy in French secondary schools. The philosophy teachers are, it appears, allowed the greatest freedom both as to method and as to subject, and most of them concentrate on instruction "not so much in facts or ideas as in the sovereign art of passing judgment". 'They seek to equip their pupils with the techniques of self-knowledge and of objective thinking. To them is available the sole opportunity given to secondary school teachers of "effectively helping their pupils to decide for themselves with their own reasoning powers, the use they are to make of their freedom as citizens".

\section{The Differential Analyser in Electrical Engineering}

$\mathrm{I}^{\mathrm{N}}$ recent years, there has been a considerable development in the design and use of mechanical and electrical instruments for carrying out calculations of various kinds. These calculations can be irvided into two main groups : first, there are those which consist of the four processes of arithmetic (addition, subtraction, multiplication and division) combined in any way, and secondly there are those which involve the further ideas of the calculus. For the latter group of calculations, the solution of differential equations is one of special importance both in pure and in applied science, as in both fields there arise many kinds of differential equations which have no formal solution or none convenient for numerical evaluation, but for which quantitative information about the behaviour of the solution is required.

In a paper by Prof. D. R. Hartree and Mr. J. K. Nuttall on the differential analyser and its applications in electrical engineering ( $J$. Inst. Elec. Engineers, No. 503), a description is given of the differential analyser, and it is shown that the solutions of nonlinear differential equations can be readily obtained by its use. Although it is possible to evaluate the solutions of such equations numerically and sometimes graphical methods can be employed, yet except for simple equations, numerical methods are laborious and lengthy, and graphical methods are limited in scope and accuracy and are also laborious to carry out on a large scale. The general idea of such a machine was due to Lord Kelvin. The practical realization of the idea is due to Dr. V. Bush, of the Massachusetts Institute of Technology, where the first machine of this kind was designed and construoted. A similar machine built by the MetropolitanVickers Electrical Co., Ltd., is installed at the University of Manchester and there is another at the University of Pennsylvania.

Immediately following the paper describing the differential analyser, there is an excellent account of the practical application of it to study the transients on a distortionless transmission line, by Prof. Hartree and Dr. Porter. The ever-increasing volume of technical literature on lightning and its effects on transmission lines and terminal equipment provides evidence of the attention now being paid by engineers to this problem. It is now known that the severity of a transient occurring at a line terminal may be lessened by flattening the front of the wave, by means of wave modifiers or surge absorbers or by reducing its amplitude by means of 'arresters' of various types. The principal features of the majority of arresters of the 'spillover' type is that their action depends on the use of a material having a non-linear current-voltage characteristic.

This paper describes the solution of the problem of evaluating the history of a transient on a finite line with such a non-linear impedance at one or both ends. The behaviour of a transient on a finite distortionless line can be described in terms of two travelling-wave systems propagated in opposite directions along the line. The voltages in these two waves satisfy two ordinary differential equations depending on the terminal conditions, and involving a relation between the voltage at two times differing by the time of travel of a wave from one end of the line to the other and back. Equations of this type can be handled mechanically by means of the differential analyser, and the use of the differential analyser does not require that the terminal impedances should have linear characteristics. The authors apply the instrument to a line with a capacitance at one end and a non-linear resistance (lightning arrester) at the other. Specimens of machine solutions, both with a linear and a non-linear resistance at the far end, are given and discussed, and compared with oscillograms taken on an actual line. 